\title{
Asociación entre niveles de TNF- $\alpha$ en fluido crevicular gingival de dientes con periodontitis apical asintomática
}

\author{
Levels of TNF- $\alpha$ increase in gingival crevicular fluid of teeth with \\ asymptomatic apical periodontitis
}

\author{
Garrido Flores $\mathrm{M}^{1}$, Ordenes Vitali $\mathrm{T}^{2}$, Segú Cabrera $\mathrm{C}^{3}$, Baeza Paredes $\mathrm{M}^{4}$, \\ García-Sesnich $\mathrm{J}^{5}$, Hernández Ríos $\mathrm{M}^{6}$
}

\begin{abstract}
RESUMEN
Introducción: Como consecuencia de necrosis pulpar séptica, el ingreso de productos bacterianos en el periápice induce la producción de citoquinas pro-inflamatorias como el Factor de Necrosis Tumoral (TNF)- $\alpha$, que entre otras funciones participa en la diferenciación y activación de los osteoclastos para inducir reabsorción ósea, fenómeno característico de la periodontitis apical asintomática (PAA). El fluido crevicular gingival (FCG) ofrece un gran potencial como fuente de factores asociados con la actividad osteoclástica. El objetivo del presente estudio fue determinar los niveles de TNF- $\alpha$ en FCG de dientes con PAA y controles sanos contralaterales. Métodos: Se incluyeron 14 pacientes en la Clínica de Endodoncia de la Facultad de Odontología de la Universidad de Chile con diagnóstico clínico de PAA y se obtuvieron muestras de FCG con tiras de papel a partir de los dientes afectados y sanos contralaterales por un periodo estandarizado de 30 segundos. Se determinaron las concentraciones de proteínas totales mediante el método del ácido bisciconitico y los niveles de TNF- $\alpha$, mediante ensayo ELISA. Los datos se analizaron con Test-t pareado utilizando el programa StataV11. Resultados: Se detectaron niveles de TNF- $\alpha$ significativamente mayores en el FCG de dientes con PAA estandarizados, tanto por 30 segundos de toma de muestra como por mg de proteínas totales. Conclusiones: Este estudio provee evidencia preliminar de que los niveles de TNF- $\alpha$ en el FCG reflejan la presencia de PAA y podría ser de utilidad como complemento al diagnóstico clínico y monitoreo del estado de salud o enfermedad de los tejidos perirradiculares.
\end{abstract}

Rev. Clin. Periodoncia Implantol. Rehabil. Oral Vol. 4(3); 130-133, 2011.

Palabras clave: TNF- $\alpha$, periodontitis apical asintomática, fluido crevicular gingival.

\section{ABSTRACT}

Introduction: As a consequence of septic pulp necrosis, the entry of bacterial products into periapical tissues induces the release of pro-inflammatory cytokines, such as the tumor necrosis factor (TNF)- $\alpha$. This pleiotropic cytokine is involved in the differentiation and activation of osteoclasts to induce bone resorption, a hallmark of asymptomatic apical periodontitis (AAP). Gingival crevicular fluid (GCF) has a great potential as a source of factors associated with osteoclastic activity. The aim of this study was to determine the levels of TNF- $\alpha$ in GCF of teeth with PAA and contralateral healthy controls. Methods: A total of 14 patients with clinical diagnosis of AAP were enrolled from the Clinic of Endodontics, Faculty of Dentistry, Universidad de Chile during 2009 and 30-second GCF samples were obtained with paper strips from AAP teeth and contralateral healthy controls. Total protein concentration and TNF- $\alpha$ levels were determined through bisciconitic acid method and ELISA assay, respectively. Paired $t$ test and StataV11 software were used for statistical analysis. Results: Levels of TNF- $\alpha$ were significantly higher in GCF from teeth with AAP than controls when standardized by either 30 s of sampling and total protein content. Conclusions: The present study provides preliminary data supporting that TNF- $\alpha$ levels in GCF reflect periapical status. Screening of TNF- $\alpha$ levels in GCF might represent a useful side-diagnostic tool to the monitoring of the apical status.

Rev. Clin. Periodoncia Implantol. Rehabil. Oral Vol. 4(3); 130-133, 2011.

Key words: TNF- $\alpha$, asimptomatic apical periodontitis, gingival crevicular fluid.

\section{INTRODUCCIÓN}

La Periodontitis Apical Asintomática (PAA) consiste en la inflamación y destrucción de los tejidos perirradiculares en ausencia de sintomatología clínica, que se refleja radiográficamente como un área radiolúcida periapical, causada por el encuentro dinámico entre agentes de etiología infecciosa de origen endodóntico y la respuesta inmune del hospedero(1). Actualmente, la PAA se considera como una de las causas más significativas de pérdida dentaria en adultos junto con otras enfermedades inflamatorias crónicas de las estructuras de soporte dentarias $^{(2)}$.
La PAA se caracteriza por la formación de una lesión osteolítica perirradicular que histológicamente puede corresponder a un granuloma periapical o quiste radicular inflamatorio ${ }^{(3,4)}$. Las lesiones periapicales (LPAs) se desarrollan como resultado de la activación de la respuesta inmune frente a la estimulación antigénica continua proveniente de la infección bacteriana y de los restos pulpares necróticos en los canales radiculares. Esta respuesta del hospedero involucra la producción de citoquinas proinflamatorias que inducen reabsorción ósea con la formación de tejido de granulación infiltrado por linfocitos, células plasmáticas, macrófagos, polimorfonucleares neutrófilos (PMN) y mastocitos $^{(5)}$. Entre los mediadores pro inflamatorios, el Factor de Necrosis Tumoral alfa $(\mathrm{TNF}-\alpha)$ se ha vinculado con el proceso de

1. Cirujano Dentista. Especialista en Endodoncia. Laboratorio de Biología Periodontal. Departamento de Odontología Conservadora. Facultad de Odontología, Universidad de Chile. Chile.

2. Cirujano Dentista. Especialista en Endodoncia. Facultad de Odontología, Universidad de Chile. Chile.

3. Cirujano Dentista. Especialista en Endodoncia. Facultad de Odontología, Universidad de Chile. Chile.

4. Cirujano Dentista. Laboratorio de Biología Periodontal. Facultad de Odontología, Universidad de Chile. Chile.

5. Bioquímico. Laboratorio de Biología Periodontal. Facultad de Odontología, Universidad de Chile. Chile.

6. Cirujano Dentista. Magister en Patología Oral y Candidata a Doctor en Cs. Odontológicas. Subdirectora de Investigación. Laboratorio de Biología Periodontal. Departamento de Patología. Facultad de Odontología, Universidad de Chile. Chile.

Correspondencia autor: Marcela Hernández Ríos. mhernandezrios@gmail.com. Laboratorio de Biología Periodontal. Departamento de Odontología Conservadora. Facultad de Odontología. Universidad de Chile. Sergio Livingstone Pohlhammer 943. Independencia. Santiago, Chile. Trabajo recibido el 15/09/2011. Aprobado para su publicación el 18/11/2011. Financiamiento: Proyecto FONDECYT 1090461. 
reabsorción de la matriz ósea. TNF- $\alpha$ es un mediador soluble liberado a partir de células inmunocompetentes ${ }^{(6)}$, fundamentalmente monocitos/ macrófagos. TNF- $\alpha$ tiene un amplio rango de efectos proinflamatorios e inmunomoduladores en diferentes poblaciones celulares, tales como estimular la síntesis de prostaglandinas, la producción de proteasas y activar la función osteoclástica y por tanto la reabsorción ósea. Las variadas funciones de TNF- $\alpha$ sugieren que éste desempeña un papel importante en la mediación de las respuestas inmunoinflamatorias iniciadas por infección o injuria ${ }^{(1,7)}$. Se han descrito niveles elevados de TNF- $\alpha$ en un modelo experimental de lesiones periapicales inducidas en ratas en asociación con la expansión de la lesión osteolítica; además TNF- $\alpha$ actúa en forma sinérgica con otros mediadores pro-osteolíticos como el ligando del receptor del factor nuclear kappa B (RANKL) durante la osteclastogénesis ${ }^{(8)}$. En línea con estos estudios, se ha determinado que los niveles de TNF- $\alpha$ se encuentran aumentados en muestras de homogeneizados de lesiones periapicales, ya sea sintomáticas o asintomáticas, comparados con tejido periodontal de pacientes sanos ${ }^{(9)}$. De este modo, la infección del tejido periapical es capaz de promover la expresión de mediadores proinflamatorios y la reabsorción ósea subsecuente ${ }^{(10)}$.

El fluido crevicular gingival (FCG) constituye un extravasado plasmático que puede obtenerse de forma no invasiva desde el surco gingival. Está constituido por moléculas derivadas del suero, células hospederas del periodonto, leucocitos y productos bacterianos provenientes de la placa subgingival. En condiciones inflamatorias, este fluido contiene componentes derivados de los procesos de destrucción local de la matriz extracelular y ofrece un gran potencial como una fuente de factores que pueden asociarse con la actividad osteoclástica y el estado de salud o enfermedad de los tejidos periodontales. En un estudio preliminar se observaron cambios en los niveles de metaloproteinasas de matriz (MMP) -9 y detección de MMP-2 en FCG en asociación con la presencia de lesiones periapicales, apoyando el análisis de este fluido como potencial método complementario al monitoreo clínico de estas lesiones; sin embargo, se desconocen las variaciones en otros mediadores claves como TNF- $\alpha$ a nivel del FCG ${ }^{(11)}$.

Considerando el importante rol que desarrollan las citoquinas proinflamatorias, particularmente TNF- $\alpha$, en los procesos osteolíticos de lesiones periapicales y la potencial aplicación del análisis del FCG como método no invasivo en la evaluación y monitoreo del estado de salud o enfermedad de estas lesiones, el propósito del presente estudio fue determinar los niveles de TNF- $\alpha$ en FCG de dientes con PAA y controles sanos contralaterales ${ }^{(1)}$.

\section{MATERIALES Y MÉTODO}

\section{Pacientes}

En el presente estudio de casos y controles, los pacientes fueron seleccionados de la Clínica de Endodoncia de la Escuela de Graduados de la Facultad de Odontología de la Universidad de Chile durante el año 2009 por un odontólogo especialista en endodoncia calibrado. El tamaño muestral se determinó con una potencia estadística $90 \%$. En base a esto, se seleccionaron 14 sujetos con diagnóstico clínico de PAA según los criterios definidos previamente ${ }^{(12)}$ en dientes uni o multirradiculares con formación radicular completa, evidenciada por la presencia de lesión apical radiográfica $>3 \mathrm{~mm}$ en dientes con caries extensa y pruebas clínicas de sensibilidad pulpar negativas. Los dientes incluidos no presentaban sintomatología dolorosa. Los criterios de exclusión fueron presencia de trauma oclusal, enfermedades periodontales, enfermedad sistémica y uso de antibióticos y antiinflamatorios no esteroidales en los últimos 6 meses previos al estudio. A partir de los sujetos seleccionados (edad promedio de $49.5 \pm 13.9$ años, 6 pacientes eran mujeres) se obtuvieron muestras de FCG de los dientes con PAA y de dientes sanos contralaterales como controles. El protocolo fue claramente explicado por los investigadores a todos los participantes del estudio, los que firmaron el consentimiento informado aprobado por el comité de ética de la Facultad de Odontología de la Universidad de Chile de acuerdo con los estándares éticos de la declaración de Helsinki.

\section{Colección del Fluido Crevicular Gingival (FCG)}

Las muestras de FCG se obtuvieron mediante tiras de papel (Periopaper ${ }^{\circledR}$, ProFlow, Amityville, New York, USA) desde seis sitios del diente de interés (mesiovestibular, vestibular, distovestibular, distolingual, lingual y mesiolingual), previa aislación relativa. Las tiras de papel se insertaron en el surco dentario durante un período estandarizado de 30 segundos. Luego, el fluido fue eluido en solución de $\mathrm{NaCl} 0.9 \%$ y almacenado a $-80^{\circ} \mathrm{C}$ para análisis posterior.

\section{Determinación de Concentraciones de Proteínas Totales en Muestras de FCG}

Se determinaron las concentraciones de proteínas totales en muestras de FCG, mediante el kit micro BCA (Pierce, Rockford, USA) según las indicaciones del fabricante.

\section{Determinación Niveles de TNF- $\alpha$ en FCG}

Los niveles de TNF- $\alpha$ se determinaron mediante un kit ELISA para TNF- $\alpha$ (Quantikine HS, R\&D, Minneapolis, Minnesota, EUA) siguiendo las recomendaciones del fabricante. Los niveles se obtuvieron a partir de una curva estándar y los resultados se expresaron en $\mathrm{pg} / \mathrm{ml}$ y $\mathrm{pg} / \mathrm{mg}$ de proteínas totales.

\section{Análisis Estadístico}

Debido a que los datos presentaron distribución normal, las comparaciones entre los grupos se efectuaron usando test-t pareado. Los datos se analizaron con el paquete estadístico StataV11. Se consideró significancia estadística si $p<0.05$.

\section{RESULTADOS}

Al comparar los niveles de TNF- $\alpha$ en FCG de dientes con lesiones periapicales de origen pulpar con diagnóstico clínico de PAA y controles sanos contralaterales de los mismos sujetos, expresados en valores absolutos (correspondientes a estandarización por 30 segundos de toma de muestra), se observaron niveles de TNF- $\alpha$ significativamente mayores en los dientes con PAA ( $p=0.027$; Figura 1).

En forma similar a los resultados expresados como valores absolutos, al comparar los niveles de TNF- $\alpha$ en el FCG de dientes con PAA y controles, expresados por $\mathrm{mg}$ de proteínas totales $(\mathrm{pg} / \mathrm{mg})$, se observó un aumento significativo en los niveles de TNF- $\alpha$ en el FCG de dientes con PAA ( $p=0.038$; Figura 2), mientras que la concentración de proteínas totales no varió entre ambos grupos de estudio ( $p>0.05$; Tabla 1$)$.

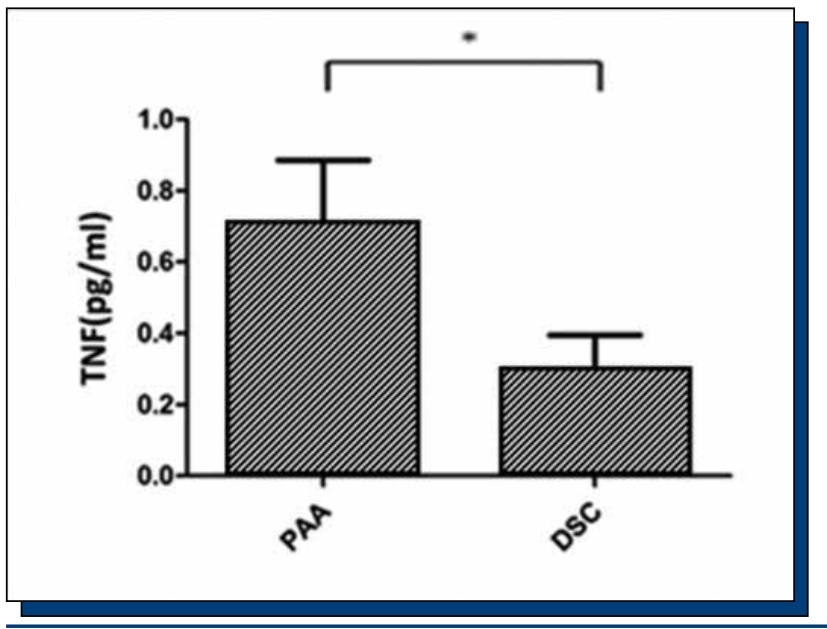

Figura 1. Niveles de TNF- $\alpha$ en FCG de dientes con periodontitis apical asintomática (PAA) y de dientes sanos contralaterales (DSC), expresados en valores absolutos ( $\mathrm{pg} / \mathrm{ml})$. ${ }^{*} \mathrm{p}=0.027$.

Tabla 1. Concentración de proteínas totales en FCG de dientes con periodontitis apical asintomática y controles.

\begin{tabular}{|c|c|c|c|}
\hline & PAA & DSC & $p$ \\
\hline CPT & $0.17 \pm 0.09$ & $0.17 \pm 0.10$ & 0.4 \\
\hline
\end{tabular}

Resultados expresados como promedio \pm desviación estándar. CPT: concentración de proteinas totales en FCG $(\mathrm{mg} / \mathrm{ml})$. PAA: dientes con periodontitis apical asintomática. DSC: dientes sanos contralaterales. 


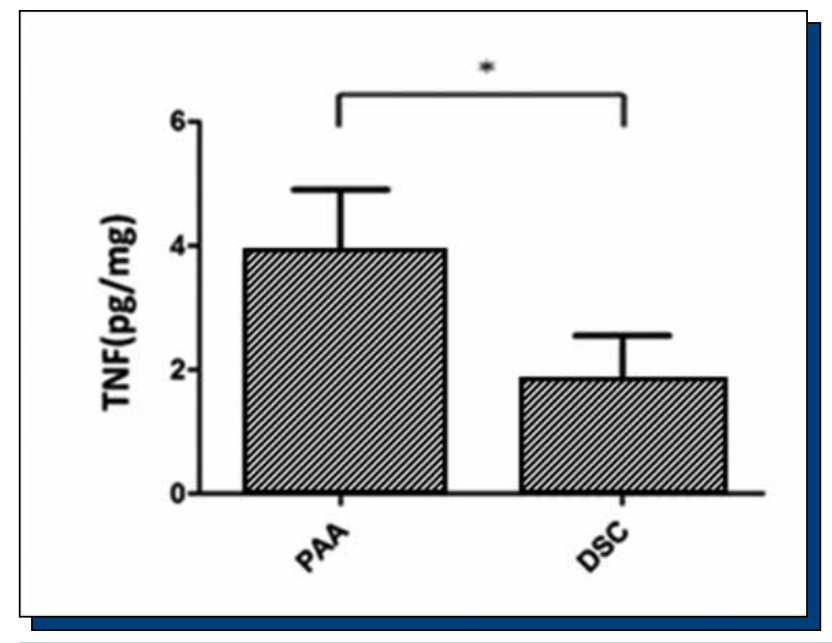

Figura 2. Niveles de TNF- $\alpha$ en FCG de dientes con periodontitis apical asintomática (PAA) y de dientes sanos contralaterales (DSC) expresados por $\mathrm{mg}$ de proteínas totales (pg/mg). ${ }^{*} \mathrm{p}=0.038$.

\section{DISCUSIÓN}

Las lesiones periapicales de origen endodóntico se caracterizan por la presencia de inflamación crónica y destrucción de los tejidos perirradiculares, tales como ligamento periodontal y hueso alveolar. La PAA ocurre habitualmente como consecuencia de necrosis pulpar séptica y la liberación de una gran variedad de mediadores pro-inflamatorios, como IL-1, IL-2, IL-6, IL-8, IL-12, TNF- $\alpha$ y RANKL, entre otros ${ }^{(13)}$. A pesar del avance progresivo en el conocimiento de la patogénesis de las lesiones perirradiculares, los eventos asociados con la destrucción de hueso periapical y el rol de las citoquinas pro-inflamatorias no se conoce completamente. Por esta razón, el diagnóstico, tratamiento y seguimiento de la PAA actualmente representan un desafío clínico. Dentro de las citoquinas involucradas en la patogénesis de estas lesiones, TNF- $\alpha$ se ha investigado en el presente estudio dados sus potentes efectos proinflamatorios, su rol clave durante la diferenciación y activación de los osteoclastos, así como por su fuerte asociación con el desarrollo y severidad de otras patologías inflamatorias crónicas, que del mismo modo que las PAA, cursan con lesiones osteolíticas como resultado de la infección de los tejido afectados ${ }^{(14-16)}$.

En el presente estudio se demostró la presencia de cambios en los niveles de TNF- $\alpha$ en FCG de dientes con PAA. Se observaron niveles mayores de TNF- $\alpha$ en FCG de los dientes afectados en comparación con controles provenientes de dientes sanos contralaterales, estandarizados tanto por 30 segundos de toma de muestra, como por mg de proteínas totales. En línea con estos resultados, en estudios previos se han demostrado aumentos de TNF- $\alpha$ en tejido de lesiones periapicales en comparación con tejido periodontal sano ${ }^{(9,17)}$. Estos datos sugieren que los niveles de TNF- $\alpha$ detectados en el FCG podrían reflejar el estado de salud-enfermedad de los tejidos periapicales.

La evaluación longitudinal de la patología periapical de origen pulpar y su respuesta al tratamiento endodóntico, se ha basado tradicionalmente en la evaluación de los parámetros clínicos y radiográficos. Sin embargo, estos métodos presentan limitaciones. EI estudio radiográfico se basa en el análisis de una imagen bidimensional que no refleja en forma confiable cambios en los tejidos perirradiculares en el corto plazo(18-22). Dadas las limitaciones de los métodos tradicionales, el análisis de biomarcadores en muestras biológicas se ha utilizado ampliamente como método complementario al diagnóstico, seguimiento y pronóstico ${ }^{(23,24)}$. Entre los tipos de muestra que se utilizan actualmente en el estudio de las lesiones periapicales, la biopsia obtenida tras una exodoncia o apicectomía del diente en cuestión, así como también el exudado periapical obtenido a través del canal radicular ${ }^{(7)}$, constituyen métodos invasivos que no permiten el seguimiento de dientes con PAA y por tanto la evaluación longitudinal de los parámetros moleculares.

En los últimos años, el FCG ha cobrado una gran importancia como potencial fuente de biomarcadores en odontología, particularmente para el estudio de las enfermedades periodontales marginales. Si bien la expresión de mediadores específicos por la concentración de proteínas totales es un método de estandarización ampliamente utilizado, en el caso particular del FCG, se recomienda la expresión de los resultados por un período estandarizado de toma de muestra de 30 segundos ${ }^{(25)}$. En la actualidad ya existen algunos dispositivos de uso en clínica para determinar experimentalmente los niveles de estos biomarcadores, particularmente para la MMP-8. Al respecto se ha visto que las variaciones en los niveles de esta enzima pueden diferenciar sujetos sanos, de sujetos con gingivitis y periodontitis, como también evaluar la respuesta al tratamiento periodontal e identificar sujetos en riesgo de progresión ${ }^{(26-}$ ${ }^{28)}$. De este modo, una herramienta no invasiva para detectar la presencia de inflamación periapical como el FCG sería de gran utilidad como complemento a la evaluación clínico-radiográfica de dientes con PAA, particularmente en aquellos casos en que la interpretación radiográfica sea incierta ${ }^{(29)}$.

El FCG constituye un extravasado plasmático con componentes derivados del catabolismo de los tejidos periodontales que puede obtenerse de forma no invasiva a partir del surco gingival. La identificación de cambios en los niveles de TNF- $\alpha$ en el FCG de dientes con PAA, sugiere que el FCG podría reflejar los procesos inflamatorios derivados del periodonto apical. El uso del FCG como potencial herramienta diagnóstica en periodontitis apical corresponde a un concepto relativamente nuevo. En línea con los resultados de este estudio, en un estudio previo ${ }^{(11)}$ se detectaron niveles significativamente mayores de MMP-9 en FCG de dientes con PAA, cuya participación es conocida en la patogenia de las periodontitis marginales y periapicales, particularmente en la degradación de la matriz orgánica del tejido óseo y tejidos blandos periodontales. Posteriormente, Burgener et al. ${ }^{(29)}$ reportaron concentraciones significativamente elevadas de proteínas totales en el FCG de dientes con lesiones apicales comparados con dientes sanos contralaterales y sugirieron la evaluación de nuevos biomarcadores específicos asociados con periodontitis apical. Si bien en el presente estudio no se encontraron diferencias significativas en la concentración de proteínas totales entre el FCG de dientes con PAA y controles, la falta de diferencias podría atribuirse a que se estudiaron procesos crónicos, que son esencialmente proliferativos; mientras que en el estudio anterior se incluyeron sujetos que consultaron en una clínica de urgencia y por tanto, estarían asociados con cuadros agudos o reagudizados que son predominantemente exudativos, determinando un aumento en la concentración de proteínas extravasadas.

En este estudio se reportaron los niveles de TNF- $\alpha$ estandarizados tanto por 30s de colección de FCG, como por proteínas totales; sin embargo, considerando los antecedentes previos, es más factible que el primero represente el método de estandarización más apropiado.

En síntesis, en el presente estudio presentamos información preliminar sobre el aumento de los niveles de TNF- $\alpha$ en FCG de dientes con PAA; sin embargo, se requiere la realización de estudios posteriores que validen la utilidad del FCG para determinar el estado de salud-enfermedad del periodonto apical, o la presencia de progresión o involución de las lesiones periapicales de origen pulpar. Esto permitiría su aplicación futura como marcador complementario al diagnóstico clínico y al monitoreo del estado de salud o enfermedad de los tejidos perirradiculares.

\section{CONFLICTOS DE INTERÉS}

Los autores descartan cualquier potencial conflicto de intereses.

\section{REFERENCIAS BIBLIOGRÁFICAS}

1. Nair PN. Pathogenesis of apical periodontitis and the causes of endodontic failures. Crit Rev Oral Biol Med, 2004; 15(6): 348-381.

2. Takahashi K. Microbiological, pathological, inflammatory, immunological and molecular biological aspects of periradicular disease. Int Endod J, 1998; 31(5): 311-325.
3. Liapatas S, Nakou M, Rontogianni D. Inflammatory infiltrate of chronic periradicular lesions: An immunohistochemical study. Int Endod J, 2003; 36(7): 464-471.

4. Ramachandran Nair PN, Pajarola G, Schroeder HE. Types and incidence of human periapical lesions obtained with extracted teeth. Oral 
Surg Oral Med Oral Pathol Oral Radiol Endod, 1996; 81(1): 93-102. 5. Matsuo T et al. Quantitative analysis of immunocompetent cells in human periapical lesions: Correlations with clinical findings of the involved teeth. J Endod, 1992; 18(10): 497-500.

6. Ataoglu $T$ et al. Interleukin-1beta and tumour necrosis factor-alpha levels in periapical exudates. Int Endod J, 2002; 35(2): 181-185.

7. Pezelj-Ribaric $S$ et al. Tumor necrosis factor-alpha in peripical tissue exudates of teeth with apical periodontitis. Mediators Inflamm, 2007; 2007: 69416 .

8. Kawashima $\mathrm{N}$ et al. Kinetics of RANKL, RANK and OPG expressions in experimentally induced rat periapical lesions. Oral Surg Oral Med Oral Pathol Oral Radiol Endod, 2007; 103(5): 707-711.

9. Prso IB et al. Tumor necrosis factor-alpha and interleukin 6 in human periapical lesions. Mediators Inflamm, 2007; 2007: 38210.

10. Chen SC et al. The upregulation of receptor activator NF-kappaB ligand expression by interleukin-1alpha and Porphyromonas endodontalis in human osteoblastic cells. Int Endod J, 2009; 42(4): 375-380.

11. Belmar MJ et al. Gelatinolytic activity in gingival crevicular fluid from teeth with periapical lesions. Oral Surg Oral Med Oral Pathol Oral Radiol Endod, 2008; 105(6): 801-806.

12. Gutmann JL et al. Identify and define all diagnostic terms for periapical/ periradicular health and disease states. J Endod, 2009; 35(12): 16581674.

13. Silva TA et al. Chemokines in oral inflammatory diseases: Apical periodontitis and periodontal disease. J Dent Res, 2007; 86(4): 306-319. 14. Zhu ZY, Liu GQ. [Changes of IL-8 and TNF-alpha in gingival crevicular fluid before and after treatment from chronic periodontitis]. Xi Bao Yu Fen Zi Mian Yi Xue Za Zhi. 26(11): 1111-1112.

15. Bastos MF et al. TNF-alpha and IL-4 levels in generalized aggressive periodontitis subjects. Oral Dis, 2009; 15(1): 82-87.

16. Javed $\mathrm{F}$ et al. Proinflammatory cytokines in the crevicular fluid of patients with peri-implantitis. Cytokine, 53(1): 8-12.

17. Fouad AF. IL-1 alpha and TNF-alpha expression in early periapical lesions of normal and immunodeficient mice. J Dent Res, 1997; 76(9): 1548-1554.
18. de Paula-Silva FW et al. Accuracy of periapical radiography and conebeam computed tomography scans in diagnosing apical periodontitis using histopathological findings as a gold standard. J Endod, 2009; 35(7): 1009-1012.

19. Patel $S$ et al. New dimensions in endodontic imaging: Part 1. Conventional and alternative radiographic systems. Int Endod J, 2009; 42(6): 447-462.

20. Trope M, Delano EO, Orstavik D. Endodontic treatment of teeth with apical periodontitis: Single vs. multivisit treatment. J Endod, 1999; 25(5): 345-350.

21. Penesis VA et al. Outcome of one-visit and two-visit endodontic treatment of necrotic teeth with apical periodontitis: A randomized controlled trial with one-year evaluation. J Endod, 2008; 34(3): 251-257.

22. Weiger R, Axmann-Krcmar D, Lost C. Prognosis of conventional root canal treatment reconsidered. Endod Dent Traumatol, 1998; 14(1): 1-9.

23. Sahingur SE, Cohen RE. Analysis of host responses and risk for disease progression. Periodontol 2000, 2004; 34: 57-83.

24. Buduneli N, Kinane DF. Host-derived diagnostic markers related to soft tissue destruction and bone degradation in periodontitis. $J$ Clin Periodontol, 2011; 38 Suppl 11: 85-105.

25. Golub LM et al. A matrix metalloproteinase inhibitor reduces bonetype collagen degradation fragments and specific collagenases in gingival crevicular fluid during adult periodontitis. Inflamm Res, 1997; 46(8): 310 319.

26. Sorsa T et al. Matrix metalloproteinases: Contribution to pathogenesis diagnosis and treatment of periodontal inflammation. Ann Med, 2006; 38(5): 306-321.

27. Mantyla $P$ et al. Monitoring periodontal disease status in smokers and nonsmokers using a gingival crevicular fluid matrix metalloproteinase-8specific chair-side test. J Periodontal Res, 2006; 41(6): 503-512.

28. Munjal SK et al. Evaluation of immunoassay-based MMP-8 detection in gingival crevicular fluid on a point-of-care platform. Ann N Y Acad Sci, 2007; 1098: 490-492.

29. Burgener B et al. Biologic markers for odontogenic periradicular periodontitis. J Endod, 36(8): 1307-1310. 